\title{
Two-Parameter Modified Ridge-Type M-Estimator for Linear Regression Model
}

\author{
Adewale F. Lukman $\mathbb{D}^{1},{ }^{1}$ Kayode Ayinde, ${ }^{2}$ B. M. Golam Kibria $\mathbb{D D}^{3}{ }^{3}$ and Segun L. Jegede \\ ${ }^{1}$ Department of Physical Sciences, Landmark University, Omu-Aran, Nigeria \\ ${ }^{2}$ Department of Statistics, Federal University of Technology, Akure, Nigeria \\ ${ }^{3}$ Department of Mathematics and Statistics, Florida International University, Miami, FL, USA
}

Correspondence should be addressed to Adewale F. Lukman; adewale.folaranmi@lmu.edu.ng

Received 9 November 2019; Revised 18 March 2020; Accepted 13 April 2020; Published 15 May 2020

Academic Editor: Roberto Rivelino

Copyright ( 92020 Adewale F. Lukman et al. This is an open access article distributed under the Creative Commons Attribution License, which permits unrestricted use, distribution, and reproduction in any medium, provided the original work is properly cited.

\begin{abstract}
The general linear regression model has been one of the most frequently used models over the years, with the ordinary least squares estimator (OLS) used to estimate its parameter. The problems of the OLS estimator for linear regression analysis include that of multicollinearity and outliers, which lead to unfavourable results. This study proposed a two-parameter ridge-type modified M-estimator (RTMME) based on the M-estimator to deal with the combined problem resulting from multicollinearity and outliers. Through theoretical proofs, Monte Carlo simulation, and a numerical example, the proposed estimator outperforms the modified ridge-type estimator and some other considered existing estimators.
\end{abstract}

\section{Introduction}

A multiple linear regression model can be defined mathematically as

$$
y=X \beta+\varepsilon,
$$

where $y$ is an $n \times 1$ vector of observations referred to as the dependent variable; $X$ is a known full column rank of $n \times p$ standardized and centered explanatory variable matrix; $\beta$ is an $p \times 1$ vector of unknown parameters; and $\varepsilon$ is an $n \times 1$ vector of disturbances with $E(\varepsilon)=0$ and dispersion matrix $\operatorname{Cov}(\varepsilon)=\sigma^{2} I, I$ is the $n \times n$ identity matrix. The ordinary least squares estimator (OLSE) of $\beta$ is given as

$$
\widehat{\beta}=\left(X^{\prime} X\right)^{-1} X^{\prime} y \text {. }
$$

According to Gauss-Markov theorem, OLS estimator is the best linear unbiased estimator (BLUE) possessing minimum variance in the class of all unbiased linear estimators [1, 2]. However, the performance of the estimator is imprecise in the presence of multicollinearity [3]. Biased estimators such as ridge regression estimator [4], Liu estimator [5] and Stein estimator [6], principal component estimator [7], modified ridge regression estimator [8], and others are often employed to tackle this problem. Another factor whose presence can negatively influence the regression coefficients of the OLS estimator is the outlier. The general practice in the literature is that one adopts robust estimators as an alternative to the OLS estimator. The M-estimator is popularly used to handle outlier in the $y$ direction [9].

Hoerl and Kennard [4] defined the ridge estimator (RE) as

$$
\widehat{\beta}(k)=\left(X^{\prime} X+k I\right)^{-1} X^{\prime} y=R_{k} \widehat{\beta},
$$

where $R_{k}=\left(X^{\prime} X+k I\right)^{-1} X^{\prime} X$ and $k>0$. However, RE can be sensitive to outliers in the $y$-direction; a remedial measure is the ridge M-estimator (RME) suggested by Silvapulle [10] as

$$
\widehat{\beta}_{M}(k)=R_{k} \widehat{\beta}_{M},
$$

where $\widehat{\beta}_{M}$ is the M-estimator of $\beta$ [11].

Dorugade [12] modified the ridge estimator and defined it as 


$$
\widehat{\beta}_{D}(k)=R_{k d 1} \widehat{\beta},
$$

where $R_{k d 1}=\left(X^{\prime} X+k d I\right)^{-1} X^{\prime} X$ and $d$ introduced as an additional basing parameter.

Following Dorugade [12], Lukman et al. [13] modified the ridge estimator in (3) and called it the modified ridgetype estimator (MRT). The estimator is defined as

$$
\widehat{\beta}_{\mathrm{MRT}}(k, d)=\left(X^{\prime} X+k(1+d) I\right)^{-1} X^{\prime} Y=R_{k d} \widehat{\beta},
$$

where $R_{k d}=\left(X^{\prime} X+k(1+d) I\right)^{-1} X^{\prime} X$.

The organization of the paper is as follows. We proposed the new estimator in Section 2 and provided a theoretical comparison among the estimators in Section 3. We discussed the robust choice of the biasing parameters in Section 4 and conducted simulation studies in Section 5 to evaluate the performance of the proposed estimator. A real-life data set is analyzed in Section 6 to illustrate the findings in the paper, and Section 7 ends with some concluding remarks.

\section{A New Estimator}

The presence of outliers in the $y$-direction affects the performance of the MRT estimator. Therefore, we suggest a ridge-type modified M-estimator (RTMME). This is defined as

$$
\widehat{\beta}_{\mathrm{RTMME}}(k, d)=R_{k d} \widehat{\beta}_{M},
$$

where $k>0,0<d<1$. It appears that $\widehat{\beta}_{\mathrm{RTMME}}(k, d)$ is a general estimator, which includes $\widehat{\beta}_{M}$ and $\widehat{\beta}_{M}(k)$ :

$$
\begin{aligned}
& \widehat{\beta}_{\text {RTMME }}(0,0)=\widehat{\beta}_{M}, \\
& \widehat{\beta}_{\text {RTMME }}(k, 0)=\widehat{\beta}_{M}(k) .
\end{aligned}
$$

The canonical form of model (1) is written as

$$
y=z \alpha+\varepsilon,
$$

where $Z=X T, \alpha=T^{\prime} \beta$, and $T$ is the orthogonal matrix whose columns contain the eigenvectors of $X^{\prime} X$. Then,

$$
Z^{\prime} Z=T^{\prime} X^{\prime} X T=\Lambda=\operatorname{diag}\left(\lambda_{1}, \lambda_{2}, \ldots, \lambda_{p}\right),
$$

where $\lambda_{1}, \lambda_{2}, \ldots, \lambda_{p}>0$ are the ordered eigenvalues of $X \prime X$.

Let $\widehat{\alpha}_{M}$ be M-defined by the solution of the M-estimating equations $\sum \varphi\left(e_{i} / s\right) z_{i}=0$, where $e_{i}=y_{i}-z_{i} \hat{\alpha}_{M}, s$ is an estimator of scale for the errors and $\varphi(\cdot)$ is some suitably chosen function [14]. The estimators presented in equations (2)-(7) can be written in canonical form as follows:

$$
\begin{aligned}
\widehat{\alpha} & =\Lambda^{-1} Z^{\prime} y, \\
\widehat{\alpha}(k) & =(\Lambda+\mathrm{kI})^{-1} Z^{\prime} y=R_{k}^{*} \widehat{\alpha}, \\
\widehat{\alpha}_{M}(k) & =R_{k}^{*} \widehat{\alpha}_{M}, \\
\widehat{\alpha}_{\mathrm{MRT}}(k, d) & =(\Lambda+k(1+d) I)^{-1} Z^{\prime} y, \\
\widehat{\alpha}_{\mathrm{MRT}}(k, d) & =R_{k d} \widehat{\alpha}, \\
\widehat{\alpha}_{\mathrm{RTMME}}(k, d) & =R_{k d} \widehat{\alpha}_{M},
\end{aligned}
$$

where $R_{k d}=\Lambda(\Lambda+k(1+d) I)^{-1}, R_{k}^{*}=\Lambda(\Lambda+k I)^{-1}$, and $k>0$.

\section{Superiority of the New Estimator}

The mean square error (MSE) criterion is used to compare the performance of the estimators. The following conditions are imposed to present our main theorems:

(i) $\varphi$ is skew-symmetric and nondecreasing

(ii) The errors are symmetric

(iii) $\Omega$ is finite

Note that any estimator of $\alpha$ has a corresponding relation $\widetilde{\beta}=T^{\prime} \widetilde{\alpha}$ such that $\operatorname{MSE}(\widetilde{\beta})=\operatorname{MSE}(\widetilde{\alpha})$. Thus, it is sufficient to consider the canonical form only. The MSEs of the aforementioned estimators are derived to be

$$
\begin{aligned}
\operatorname{MSE}(\widehat{\alpha}) & =\sigma^{2} \sum_{i=1}^{p} \frac{1}{\lambda_{i}} \\
\operatorname{MSE}\left(\widehat{\alpha}_{M}\right)= & \sum_{i=1}^{p} \Omega_{i i} \\
\operatorname{MSE}[\widehat{\alpha}(k)]= & \sigma^{2} \sum_{i=1}^{p} \frac{\lambda_{i}}{\left(\lambda_{i}+k\right)^{2}}+\sum_{i=1}^{p} \frac{k^{2} \alpha_{i}^{2}}{\left(\lambda_{i}+k\right)^{2}}, \\
\operatorname{MSE}\left[\widehat{\alpha}_{M}(k)\right]= & \sum_{i=1}^{p} \frac{\lambda_{i}^{2}}{\left(\lambda_{i}+k\right)^{2}} \Omega_{i i}+\sum_{i=1}^{p} \frac{k^{2} \alpha_{i}^{2}}{\left(\lambda_{i}+k\right)^{2}} \\
\operatorname{MSE}\left[\widehat{\alpha}_{\operatorname{MRT}}(k, d)\right]= & \sigma^{2} \sum_{i=1}^{p} \frac{\lambda_{i}}{\left(\lambda_{i}+k(1+d)\right)^{2}} \\
& +\sum_{i=1}^{p} \frac{k^{2}(1+d)^{2} \alpha_{i}^{2}}{\left(\lambda_{i}+k(1+d)\right)^{2}}, \\
\operatorname{MSE}\left[\widehat{\alpha}_{\mathrm{RTMME}}(k, d)\right]= & \sum_{i=1}^{p} \frac{\lambda_{i}^{2}}{\left(\lambda_{i}+k(1+d)\right)^{2}} \Omega_{i i} \\
& +\sum_{i=1}^{p} \frac{k^{2}(1+d)^{2} \alpha_{i}^{2}}{\left(\lambda_{i}+k(1+d)\right)^{2}},
\end{aligned}
$$

where $\Omega_{i i}=\operatorname{Cov}\left(\widehat{\alpha}_{M}\right)$.

Theorem 1. For $\operatorname{MSE}\left(\widehat{\alpha}_{R T M M E}(k, d)\right)<\operatorname{MSE}\left(\widehat{\alpha}_{M R T}(k, d)\right)$, then $\sum_{i=1}^{p} \Omega_{i i}<\sum_{i=1}^{p} \sigma^{2} \lambda_{i}^{-1}$ for every $k>0$ and $i=1,2, \ldots, p$, where $\Omega_{i i}$ are the diagonal elements of $\Omega$.

Proof. After some algebraic manipulation, the difference between $\operatorname{MSE}\left(\widehat{\alpha}_{\mathrm{RTMME}}(k, d)\right)-\operatorname{MSE}\left(\widehat{\alpha}_{\mathrm{MRT}}(k, d)\right)$ gives

$$
\begin{gathered}
\Delta_{1}=\operatorname{MSE}\left(\widehat{\alpha}_{\text {RTMME }}(k, d)\right)-\operatorname{MSE}\left(\widehat{\alpha}_{M R T}(k, d)\right), \\
\Delta_{1}=\sum_{i=1}^{p} \frac{\lambda_{i}^{2} \Omega_{i i}-\sigma^{2} \lambda_{i}}{\left(\lambda_{i}+k(1+d)\right)^{2}} .
\end{gathered}
$$


For equation (14) to be less than zero, we should have $\Omega_{i i}-\sigma^{2} \lambda_{i}^{-1}<0$, which also implies $\sum_{i=1}^{p} \Omega_{i i}<\sum_{i=1}^{p} \sigma^{2} \lambda_{i}^{-1}$ for $k>0,0<d<1$ and for $i=1,2, \ldots, p$.
Theorem 2. When $\operatorname{MSE}\left(\widehat{\alpha}_{R T M M E}(k, d)\right)<\operatorname{MSE}\left(\widehat{\alpha}_{M}(k)\right)$, there exists a positive constant $k>k_{i}>0$, where

$$
k_{1 i}=\frac{\lambda_{i}\left[\sqrt{\left((d+2)\left(\alpha_{i}^{2}-\Omega_{i i}\right)\right)^{2}+8 \alpha_{i}^{2} \Omega_{i i}(d+1)}-(d+2)\left(\alpha_{i}^{2}-\Omega_{i i}\right)\right]}{4 \alpha_{i}^{2}(d+1)} .
$$

Proof. The difference MSE $\left(\widehat{\alpha}_{\mathrm{RTMME}}(k, d)\right)-\operatorname{MSE}\left(\widehat{\alpha}_{M}(k)\right)$ is :

$$
\Delta_{2}=\sum_{i=1}^{p} \frac{\lambda_{i}^{2} \Omega_{i i}+k^{2}(1+d)^{2} \alpha_{i}^{2}}{\left(\lambda_{i}+k(1+d)\right)^{2}}-\sum_{i=1}^{p} \frac{\lambda_{i}^{2} \Omega_{i i}+k^{2} \alpha_{i}^{2}}{\left(\lambda_{i}+k\right)^{2}} .
$$

The difference is strictly less than zero if and only if, after simplification, the following expression holds:

$$
k^{2}\left[2 \alpha_{i}^{2} \lambda_{i} d\right]+k\left[\lambda_{i}^{2} d(d+2)\left(\alpha_{i}^{2}-\Omega_{i i}\right)\right]-2 \Omega_{i i} \lambda_{i}^{3} d<0 .
$$

Solving inequality (17) for $k$, we get

$$
k_{1 i}=\frac{\lambda_{i}\left[\sqrt{\left((d+2)\left(\alpha_{i}^{2}-\Omega_{i i}\right)\right)^{2}+8 \alpha_{i}^{2} \Omega_{i i}(d+1)}-(d+2)\left(\alpha_{i}^{2}-\Omega_{i i}\right)\right]}{4 \alpha_{i}^{2}(d+1)} .
$$

Notice that if $\lambda_{i}\left[\sqrt{\left((d+2)\left(\alpha_{i}^{2}-\Omega_{i i}\right)\right)^{2}+8 \alpha_{i}^{2} \Omega_{i i}(d+1)}\right.$ $\left.-(d+2)\left(\alpha_{i}^{2}-\Omega_{i i}\right)\right]>0 ; k_{1 i}>0$, and there is, therefore, a positive constant $k>k_{1 i}>0$.

Theorem 3. A necessary condition for $\operatorname{MSE}\left(\widehat{\alpha}_{R T M M E}(k, d)\right)$ $<\operatorname{MSE}\left(\widehat{\alpha}_{M}\right)$ is

$$
\sum_{i=1}^{p} \Omega_{i i}>\sum_{i=1}^{p} \frac{k(1+d) \alpha_{i}^{2}}{2 \lambda_{i}+k(1+d)}
$$

Proof. The difference $\operatorname{MSE}\left(\widehat{\alpha}_{\mathrm{RTMME}}(k, d)\right)-\operatorname{MSE}\left(\widehat{\alpha}_{M}\right)$ is

$$
\begin{aligned}
& \Delta_{3}=\sum_{i=1}^{p} \frac{\lambda_{i}^{2} \Omega_{i i}+k^{2}(1+d)^{2} \alpha_{i}^{2}}{\left(\lambda_{i}+k(1+d)\right)^{2}}-\sum_{i=1}^{p} \Omega_{i i}, \\
& \Delta_{3}=-\Omega_{i i}\left(2 \lambda_{i} k(1+d)+k^{2}(1+d)^{2}\right)+k^{2}(1+d)^{2} \alpha_{i}^{2} .
\end{aligned}
$$

To obtain $\operatorname{MSE}\left(\widehat{\alpha}_{\mathrm{RTMME}}(k, d)\right)<\operatorname{MSE}\left(\widehat{\alpha}_{M}\right)$,

$$
\sum_{i=1}^{p} \Omega_{i i}<\sum_{i=1}^{p} \frac{k(1+d) \alpha_{i}^{2}}{2 \lambda_{i}+k(1+d)}
$$

Theorem 2 provided $k>k_{1 i}$ such that $\operatorname{MSE}\left(\widehat{\alpha}_{\mathrm{RTMME}}\right.$ $(k, d))<\operatorname{MSE}\left(\widehat{\alpha}_{M}\right)$. Besides this, in the Theorem of Silvapulle [10] (part (i), pg. 321), it is indicated that there exists $k>0$ such that $\operatorname{MSE}\left(\widehat{\alpha}_{M}(k)\right)-\operatorname{MSE}\left(\widehat{\alpha}_{M}\right)<0$. Thus, we obtain the corollary as follows.

Corollary. There exists $k>0$ such that $\operatorname{MSE}\left(\widehat{\alpha}_{R T M M E}(k, d)\right)$ $-\operatorname{MSE}\left(\widehat{\alpha}_{M}\right)$.

\section{Robust Choice of $\boldsymbol{k}$ and $\boldsymbol{d}$ for $\widehat{\alpha}_{\text {RTMME }}(k, d)$}

For the robust biasing parameters $k$ and $d$ for the modified two-parameter estimator, the optimal values can be determined by minimizing equation (23) with respect to each of the parameters:

$$
f(k, d)=\sum_{i=1}^{p} \frac{\lambda_{i}^{2}}{\left(\lambda_{i}+k(1+d)\right)^{2}} \Omega_{i i}+\sum_{i=1}^{p} \frac{k^{2}(1+d)^{2} \alpha_{i}^{2}}{\left(\lambda_{i}+k(1+d)\right)^{2}} .
$$

This can be obtained by solving

$$
\begin{aligned}
& \frac{\partial f(k, d)}{\partial d}=0, \\
& \frac{\partial f(k, d)}{\partial k}=0 .
\end{aligned}
$$

By doing this, we have

$$
\begin{aligned}
& d=\frac{\lambda_{i} \Omega_{i i}}{k \alpha_{i}^{2}}-1, \\
& k=\frac{\lambda_{i} \Omega_{i i}}{\alpha_{i}^{2}(1+d)} .
\end{aligned}
$$

We substitute $\Omega_{i i}$ and $\alpha_{i}^{2}$ into equations (24) and (25) with their corresponding estimates. We assume that $\widehat{\alpha}_{M}$ is normally distributed with mean $\alpha$ and covariance matrix $A^{2} \Lambda^{-1}$. This assumption holds since $n^{1 / 2}\left(\widehat{\alpha}_{M}^{2}-\alpha\right) \longrightarrow$ $N\left(0, A^{2} \Lambda^{-1}\right)$, where

$$
A^{2}=\frac{s_{o}^{2} E\left(\varphi^{2}\left(\varepsilon / s_{o}\right)\right)}{\left(E\left(\varphi^{\prime}\left(\varepsilon / s_{o}\right)\right)\right)^{2}},
$$


with the scale estimate $s_{o}$. Thus, the estimate of $\alpha_{i}^{2}$ is $\widehat{\alpha}_{M i}^{2}$, and the unbiased estimator of $\Omega_{i i}$ is asymptotically $\widehat{A}^{2} / \lambda_{i}$, where $\widehat{A}^{2}$ is given by Huber [9] as

$$
\widehat{A}^{2}=\frac{s^{2}(n-p)^{-1} \sum_{i=1}^{p}\left(\varphi\left(e_{i} / s\right)\right)^{2}}{\left(\sum_{i=1}^{p}(1 / n) \varphi^{\prime}\left(e_{i} / s\right)\right)^{2}} .
$$

We get the optimal estimator of $d$ and $k$ as

$$
\begin{aligned}
& d=\frac{\widehat{A}^{2}}{k \widehat{\alpha}_{M i}^{2}}-1, \quad i=1,2, \ldots, p, \\
& k=\frac{\widehat{A}^{2}}{(1+d) \widehat{\alpha}_{M i}^{2}}, \quad i=1,2, \ldots, p .
\end{aligned}
$$

Following Kibria [15], the arithmetic and geometric mean version of $k$ is obtained, respectively, as

$$
\begin{aligned}
& \tilde{k}_{\mathrm{GMR}}=\left[\prod_{i=1}^{p} \frac{\widehat{A}^{2}}{(1+d) \widehat{\alpha}_{M i}^{2}}\right]^{1 / p}, \\
& \tilde{k}_{\mathrm{AMR}}=\frac{1}{p} \sum_{i=1}^{p} \frac{\widehat{A}^{2}}{(1+d) \widehat{\alpha}_{M i}^{2}}
\end{aligned}
$$

The harmonic mean version is generally preferred to other versions [3]. Hence, the robust harmonic mean version of the proposed $d$ and $k$ from (31) and (32) is obtained as

$$
\begin{aligned}
\tilde{k}_{\mathrm{HMR}} & =\frac{p \widehat{A}^{2}}{\sum_{i=1}^{p}(1+d) \widehat{\alpha}_{M i}^{2}}, \\
\tilde{d}_{\mathrm{HMR}} & =\frac{p}{\sum_{i=1}^{p}(1 / d)} .
\end{aligned}
$$

The selection of the estimators of the parameters $d$ and $k$ can be obtained iteratively as follows:

Step 1: use $\widehat{d}=\min \left(\widehat{A}^{2} / \widehat{\alpha}_{M i}^{2}\right)$ to obtain an initial estimate for $d$

Step 2: from (33), get $\widetilde{k}_{\mathrm{HMR}}$ using $d$ in Step 1

Step 3: calculate $\widetilde{d}_{\mathrm{HMR}}$ in (34) by using $\widetilde{k}_{\mathrm{HMR}}$ in Step 2

Step 4: use $\widehat{d}$ in Step 1 if $\widetilde{d}_{\mathrm{HMR}}<0$

\section{Monte Carlo Simulation Study}

We adopted the simulation design by McDonald and Galarneau [16], Kibria [15], and Lukman et al. [17]. The explanatory variables are generated using the following equation:

$$
x_{i j}=\left(1-\rho^{2}\right)^{1 / 2} z_{i j}+\rho z_{i, p+1}, \quad i=1,2, \ldots, n, j=1,2, \ldots, p,
$$

where $\rho^{2}$ denotes the correlation between explanatory variables and $z_{i j}$ are pseudo-random numbers from the standard normal distribution. The coefficients $\beta_{1}, \beta_{2}, \ldots, \beta_{p}$ are selected as the normalized eigenvectors corresponding to the largest eigenvalue of $X^{\prime} X$ so that we have $\beta^{\prime} \beta=1$, which is a common restriction in simulation studies of this type ([3]). The dependent variable is then determined using

$$
y_{i}=\beta_{0}+\beta_{1} x_{i 1}+\beta_{2} x_{i 2}+\cdots+\beta_{p} x_{i p}+\varepsilon_{i}, \quad i=1,2, \ldots, n,
$$

where the error term $\varepsilon_{i}^{\prime} s$ is generated with mean and variance 0 and $\sigma^{2}$, respectively. We fixed the number of explanatory variables to three and seven $(p=3,7)$, and other parameters such as $\rho, \sigma$, and $n$ were varied; their values considered in this study are given as follows:

$$
\rho=0.7,0.8,0.9 \text { and } 0.99
$$

The standard deviation $(\sigma)$ of the error term in this simulation study is $1,5,10$

$n=20,50,100$

We considered three different cases in this study:

Case I: no outlier

Case II: one outlier

Case III: two outliers

In the case of no outliers, equation (36) is taken into consideration. For the case of one outlier, the tenth observation is changed as $y_{10}^{*}=y_{10}+20 \sigma$. For the case of two outliers, the fifth and the tenth observations are changed as $y_{5}^{*}=y_{5}+20 \sigma$ and $y_{10}^{*}=y_{10}-20 \sigma$, respectively. The experiment is replicated 2,000 times by generating new pseudo-random numbers, and the estimated MSE is calculated as

$$
\operatorname{mse}(\widehat{\alpha})=\frac{1}{2000} \sum_{j=1}^{2000}\left(\widehat{\alpha}_{i j}-\alpha_{i}\right)^{\prime}\left(\widehat{\alpha}_{i j}-\alpha_{i}\right) .
$$

The results of the simulation are presented in Tables 1-18. As expected, the OLSE is observed to have the least performance. The following observations are also made:

(i) As the error standard deviation $(\sigma)$ and the degree of multicollinearity $(\rho)$ increase, the MSEs of the estimators $\left(\alpha, \widehat{\alpha}_{M}, \widehat{\alpha}(k), \widehat{\alpha}_{M}(k), \widehat{\alpha}_{\mathrm{MRT}}\right.$, and $\left.\widehat{\alpha}_{\mathrm{RTMME}}\right)$ increase.

(ii) As the biasing parameters $k$ and $d$ increase, the MSEs of the estimators $\left(\alpha, \widehat{\alpha}_{M}, \widehat{\alpha}(k), \widehat{\alpha}_{M}(k), \widehat{\alpha}_{\mathrm{MRT}}\right.$, and $\left.\widehat{\alpha}_{\text {RTMME }}\right)$ also decrease.

(iii) The MSEs of the estimators $\left(\alpha, \widehat{\alpha}_{M}, \widehat{\alpha}(k), \widehat{\alpha}_{M}(k)\right.$, $\widehat{\alpha}_{\mathrm{MRT}}$, and $\left.\widehat{\alpha}_{\mathrm{RTMME}}\right)$ decrease as the sample size increases. However, as the number of outliers increases, the MSEs also increase.

(iv) Finally, just as in the outcome of Lukman et al. [13], the MRT estimator outperforms other estimators considered in the case of no outlier. However, when outliers were introduced, the RTMME outperforms other considered estimators. 


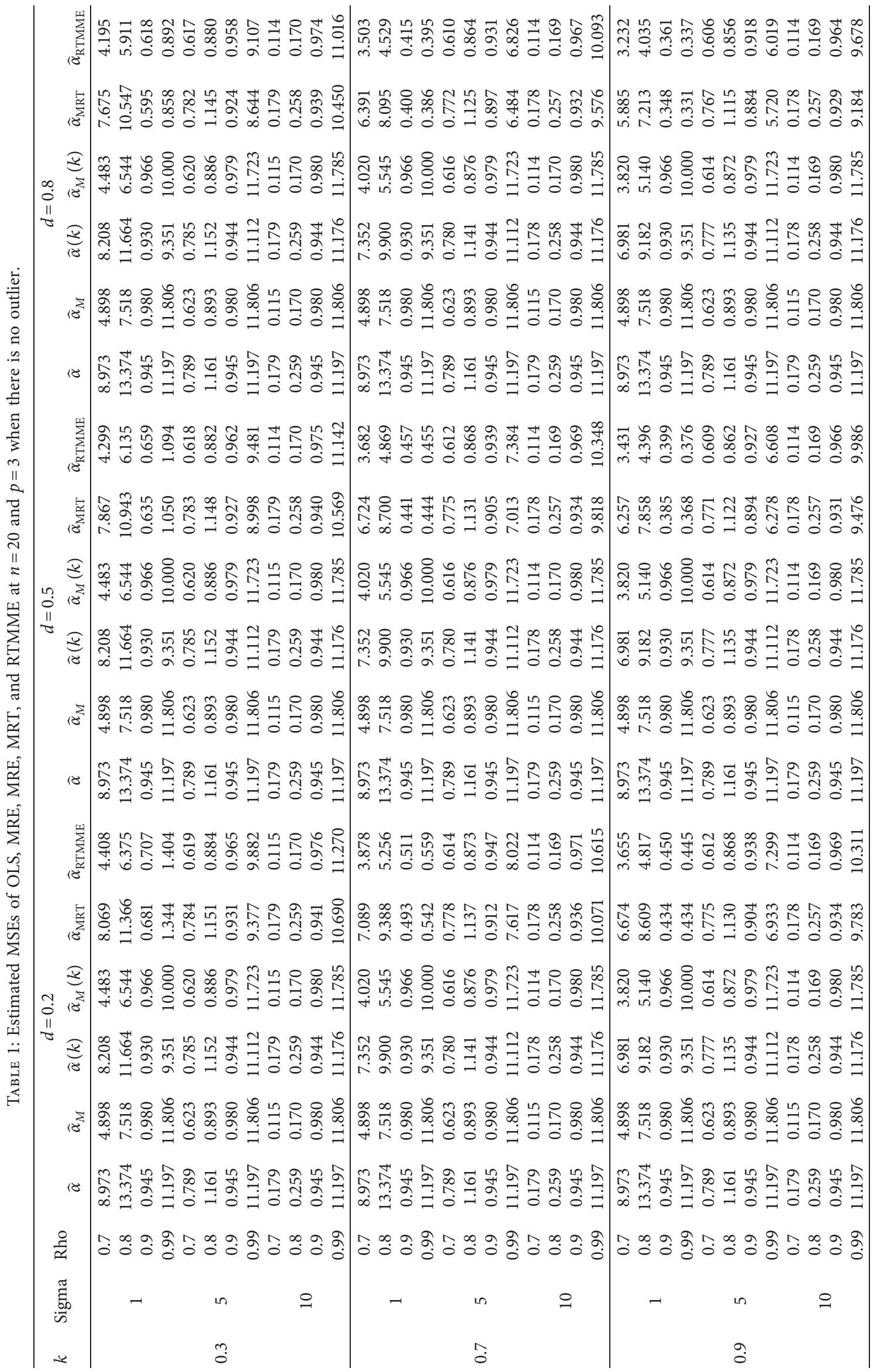




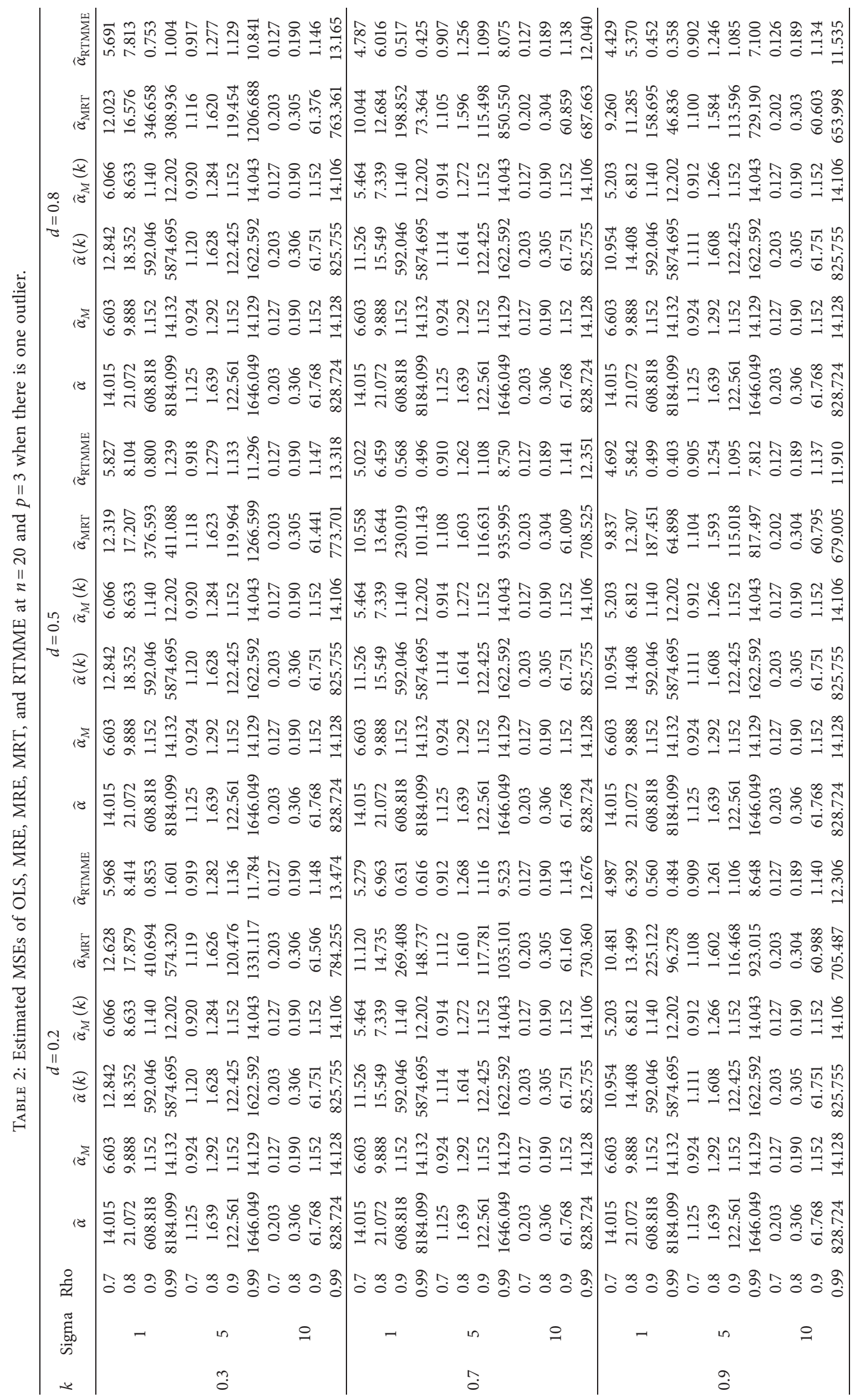




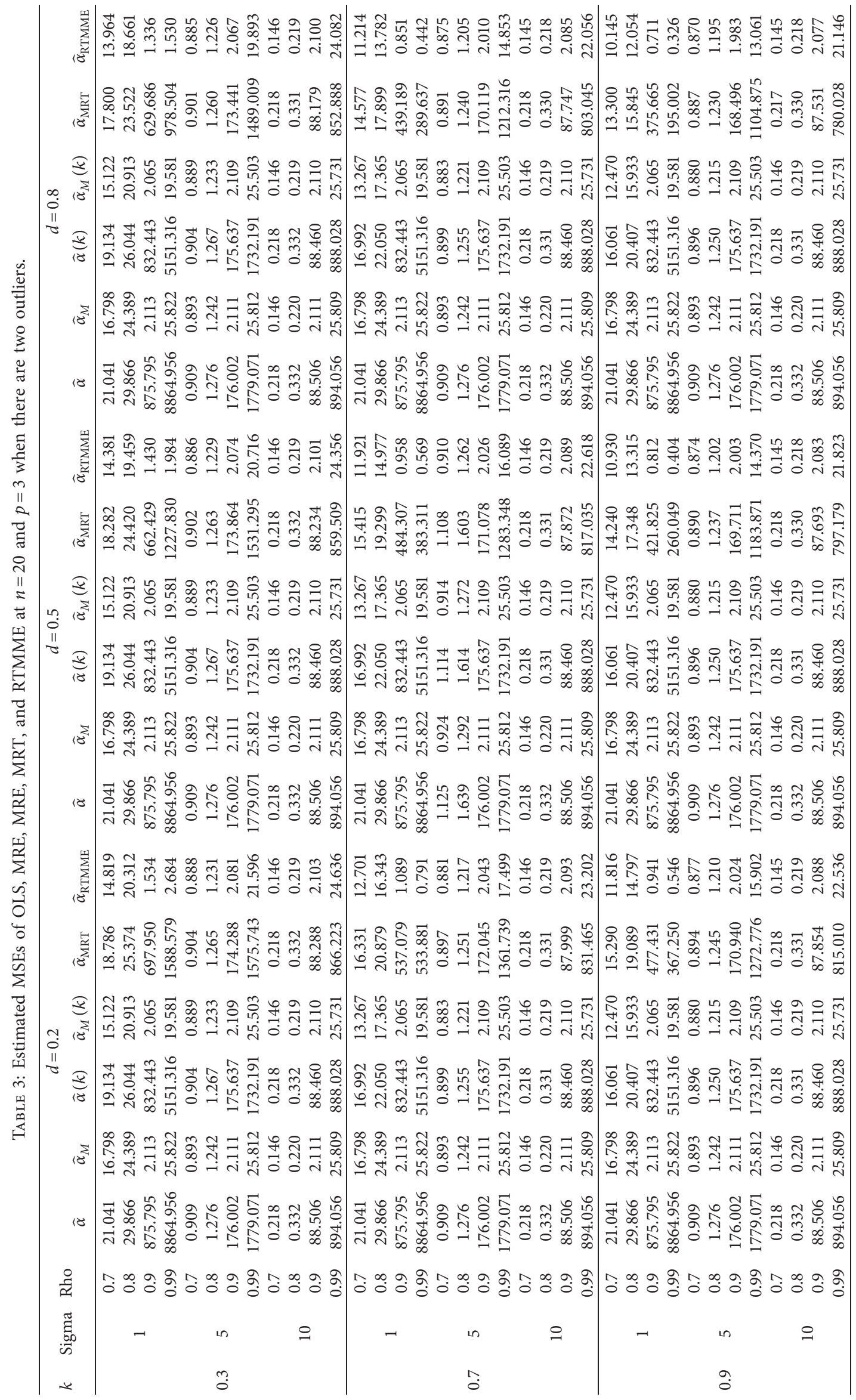




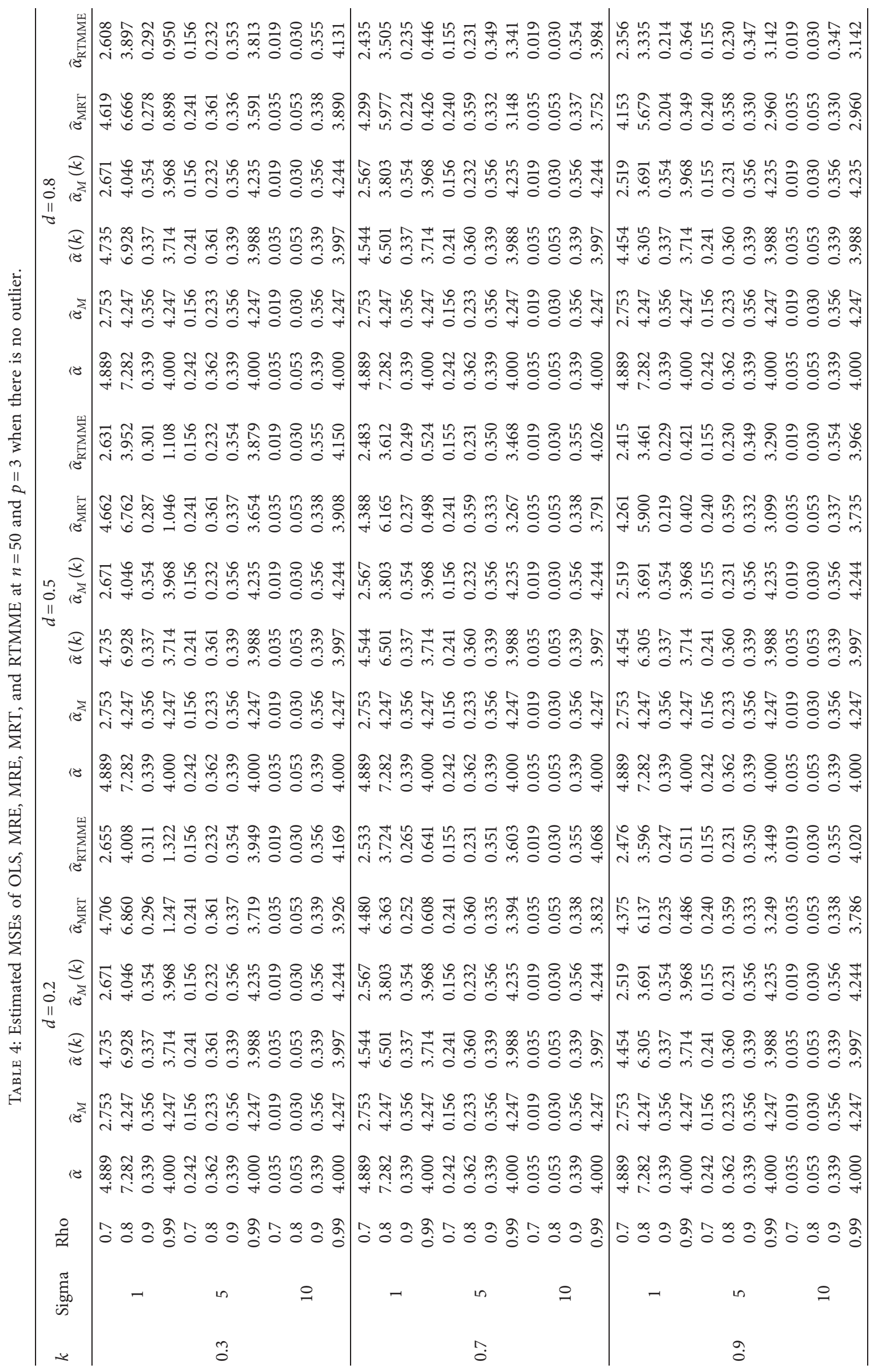




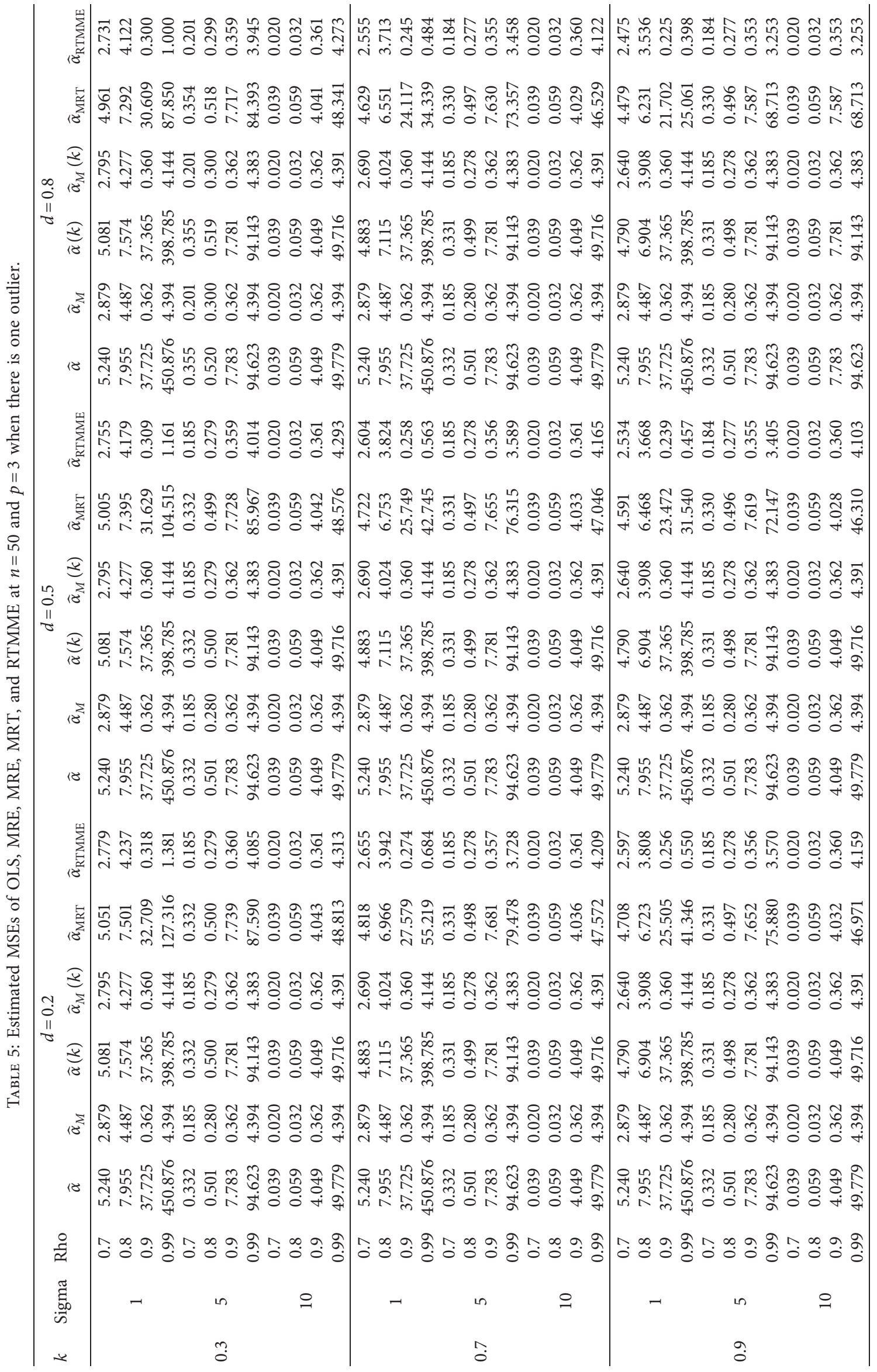




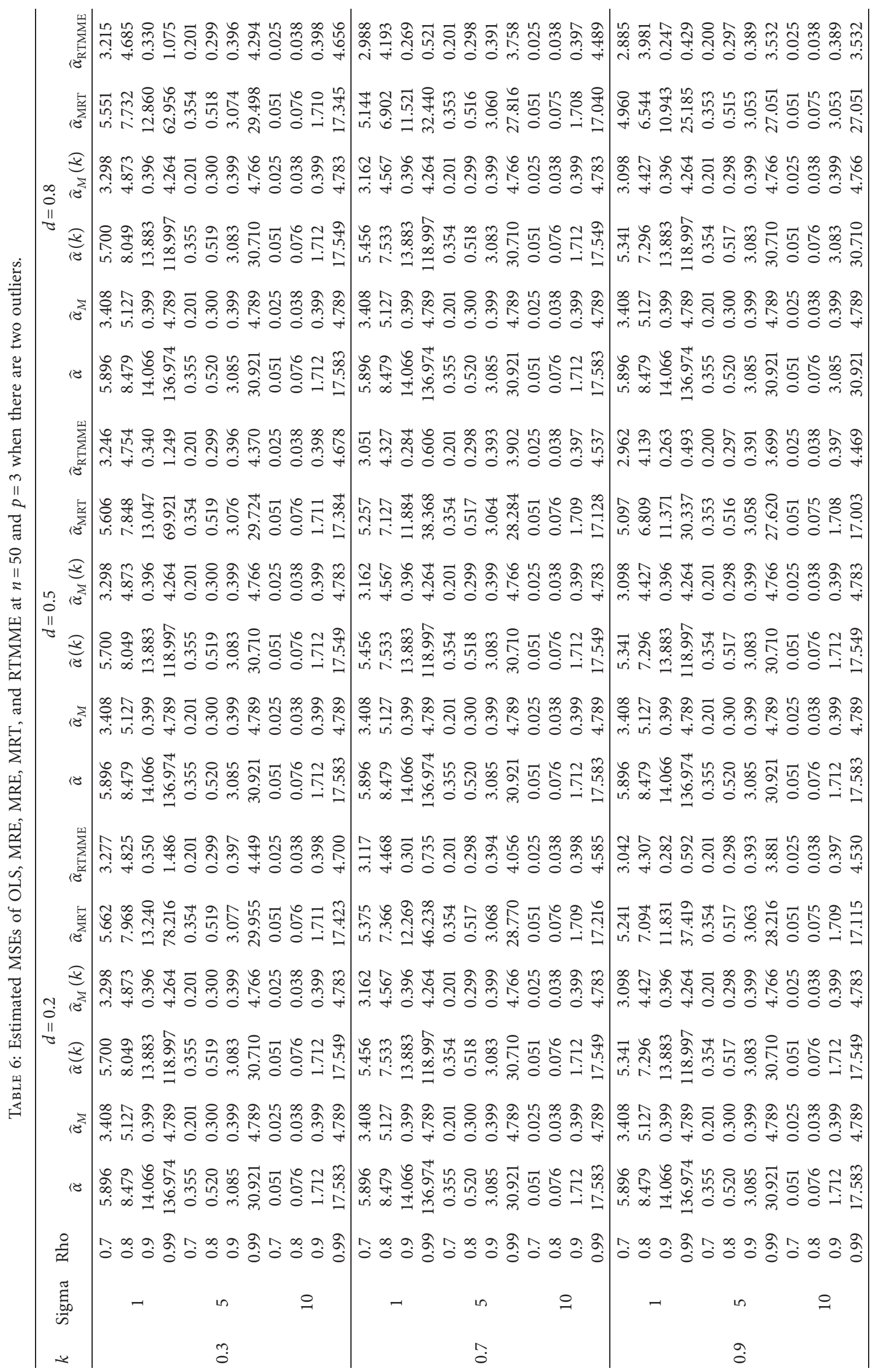




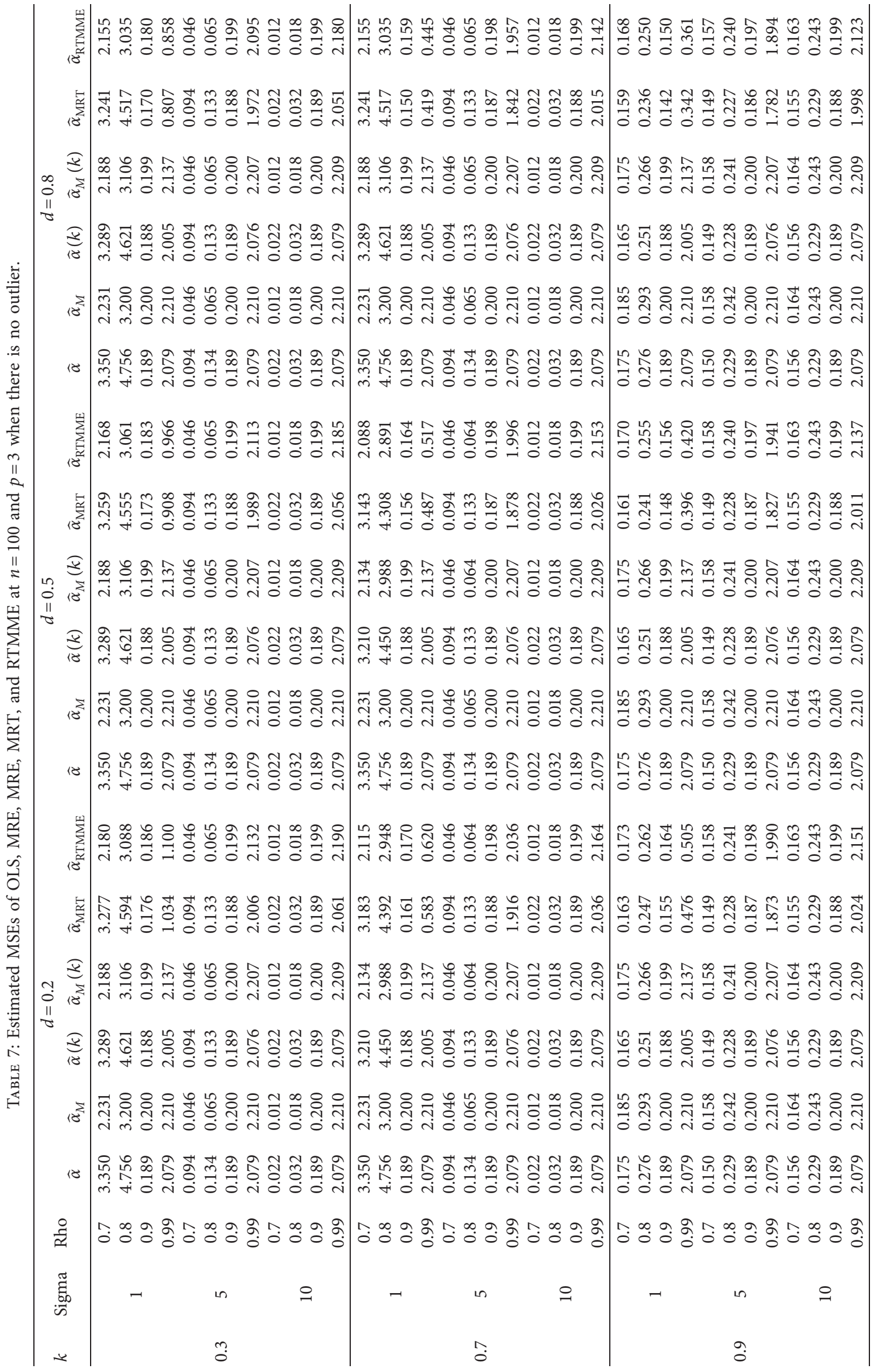




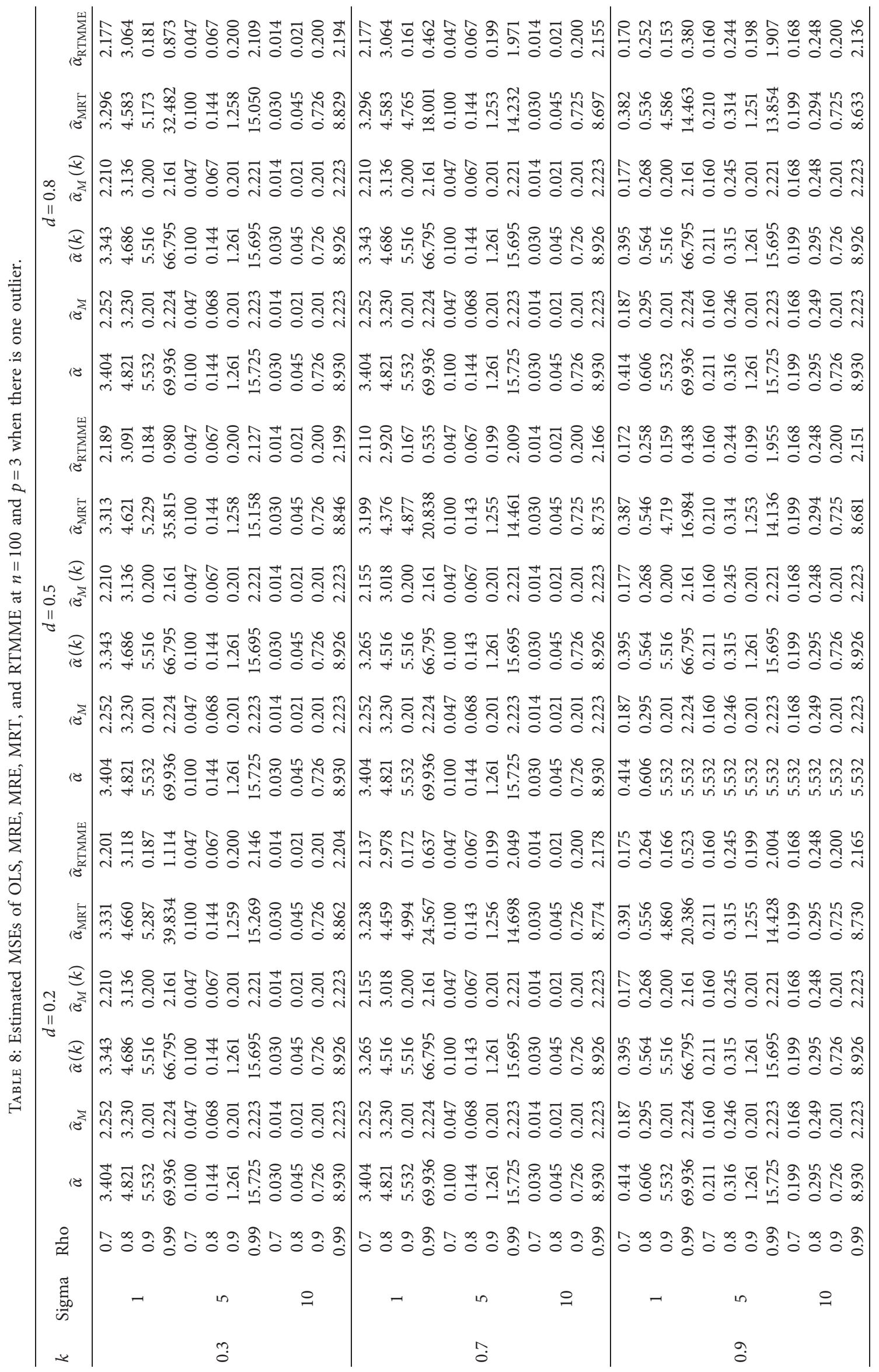




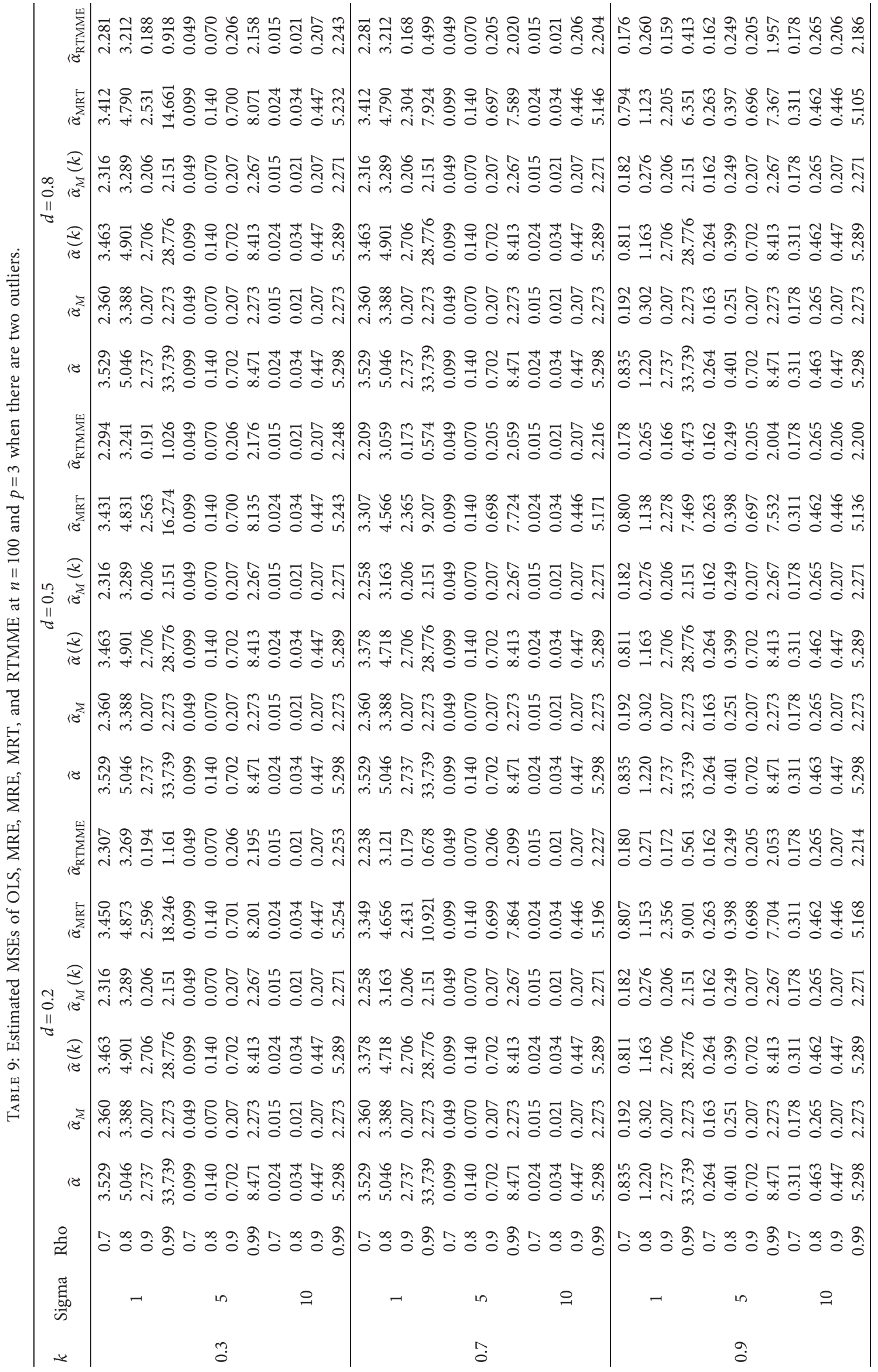




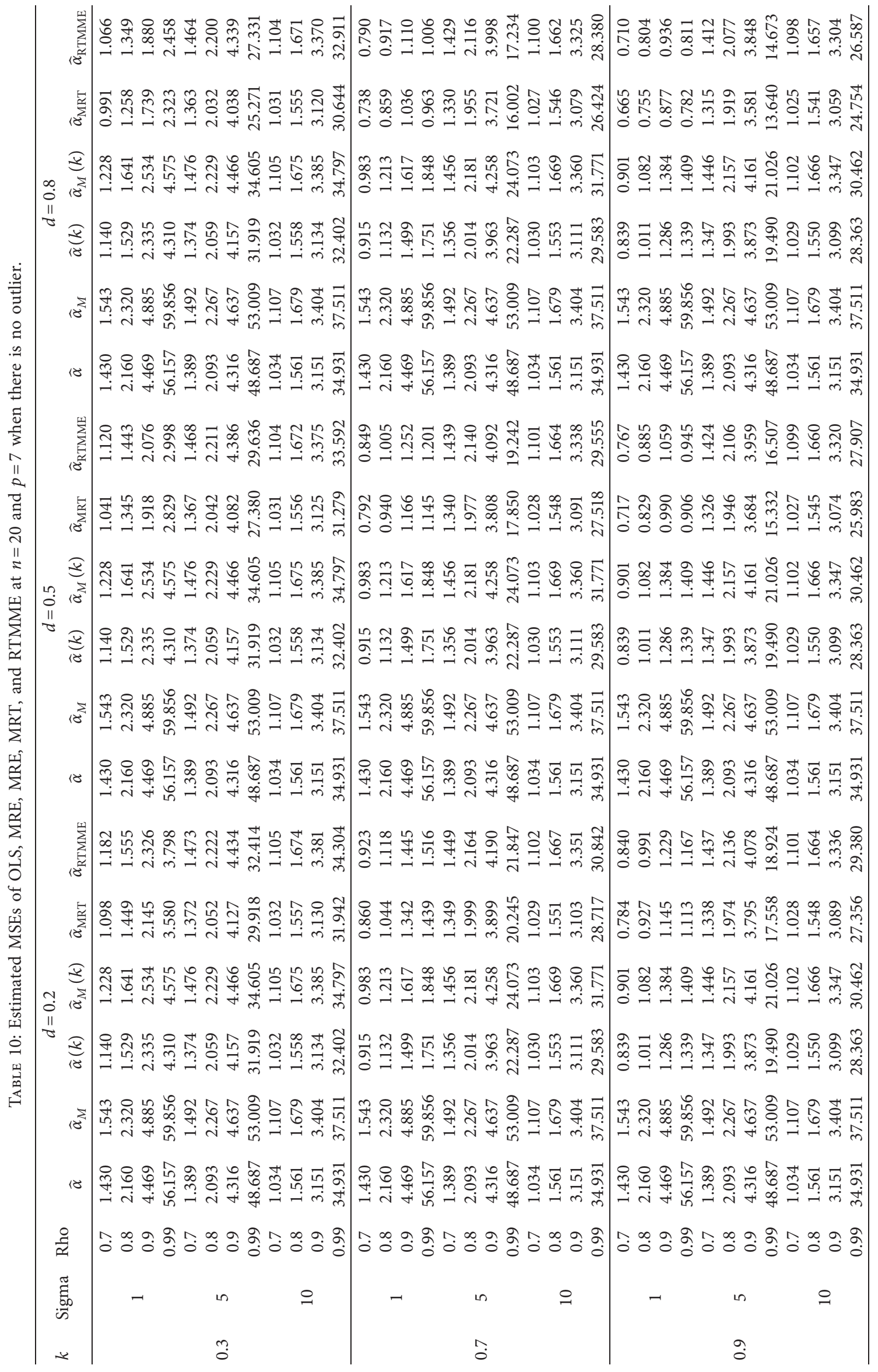




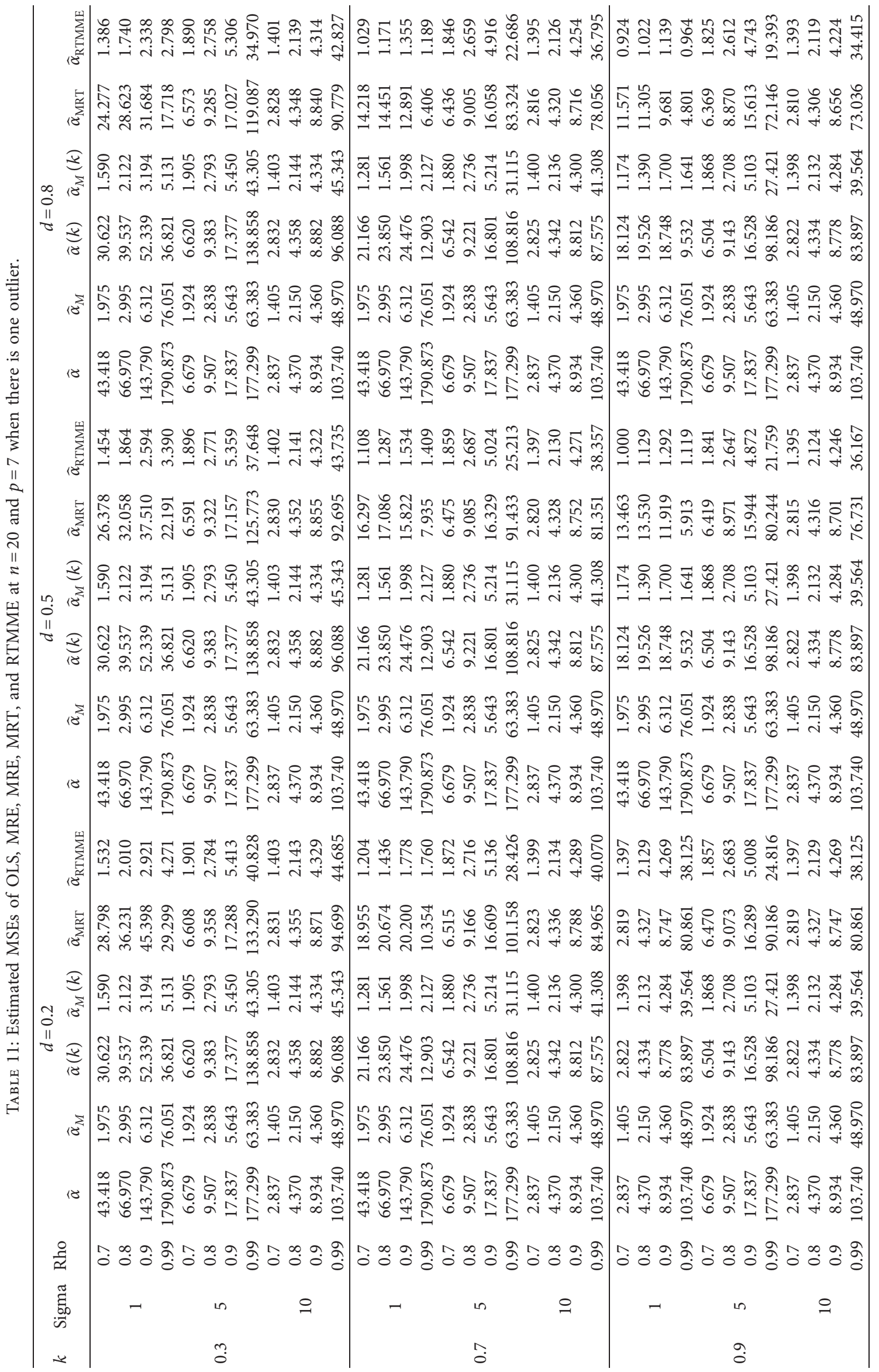




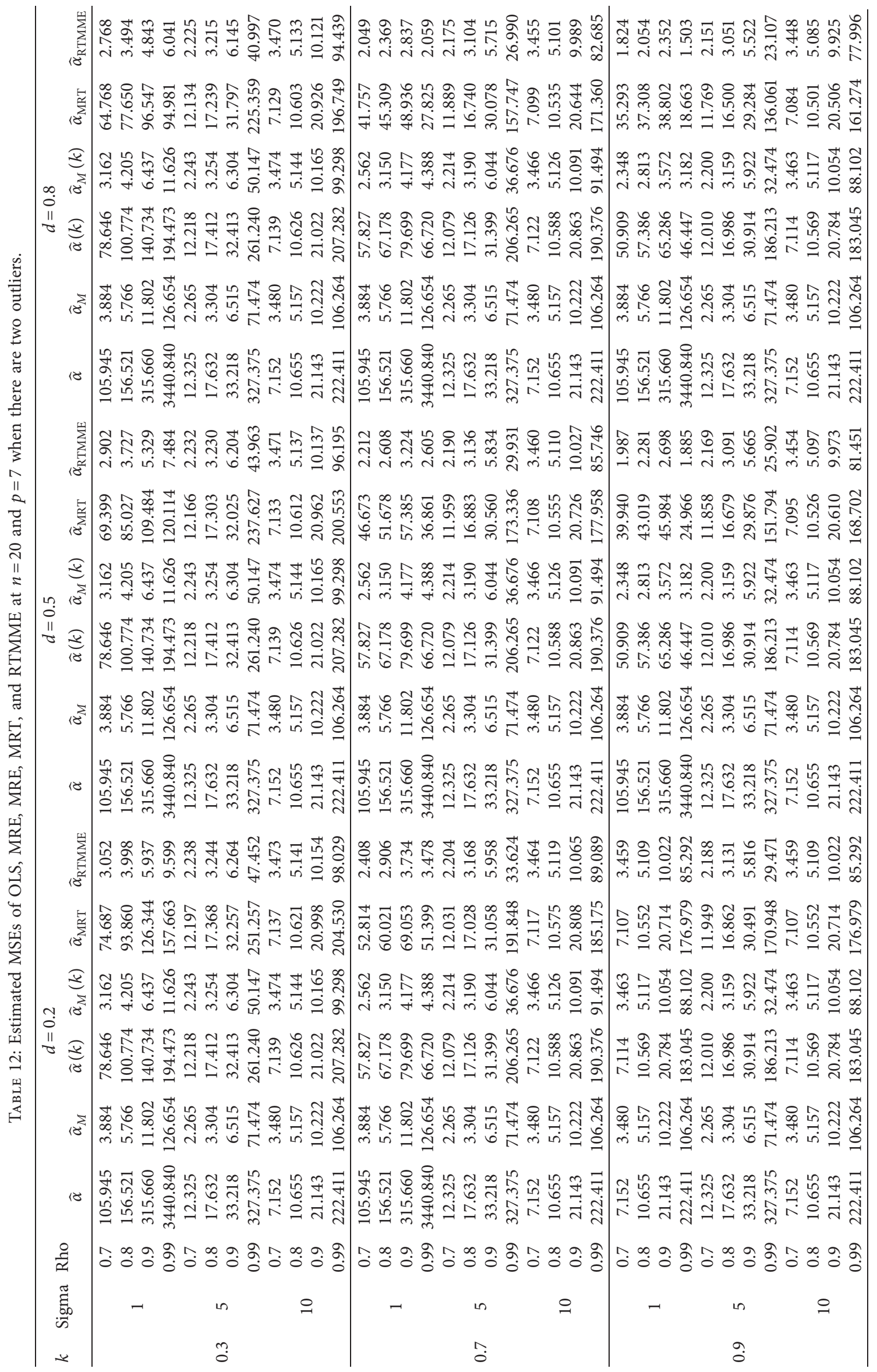




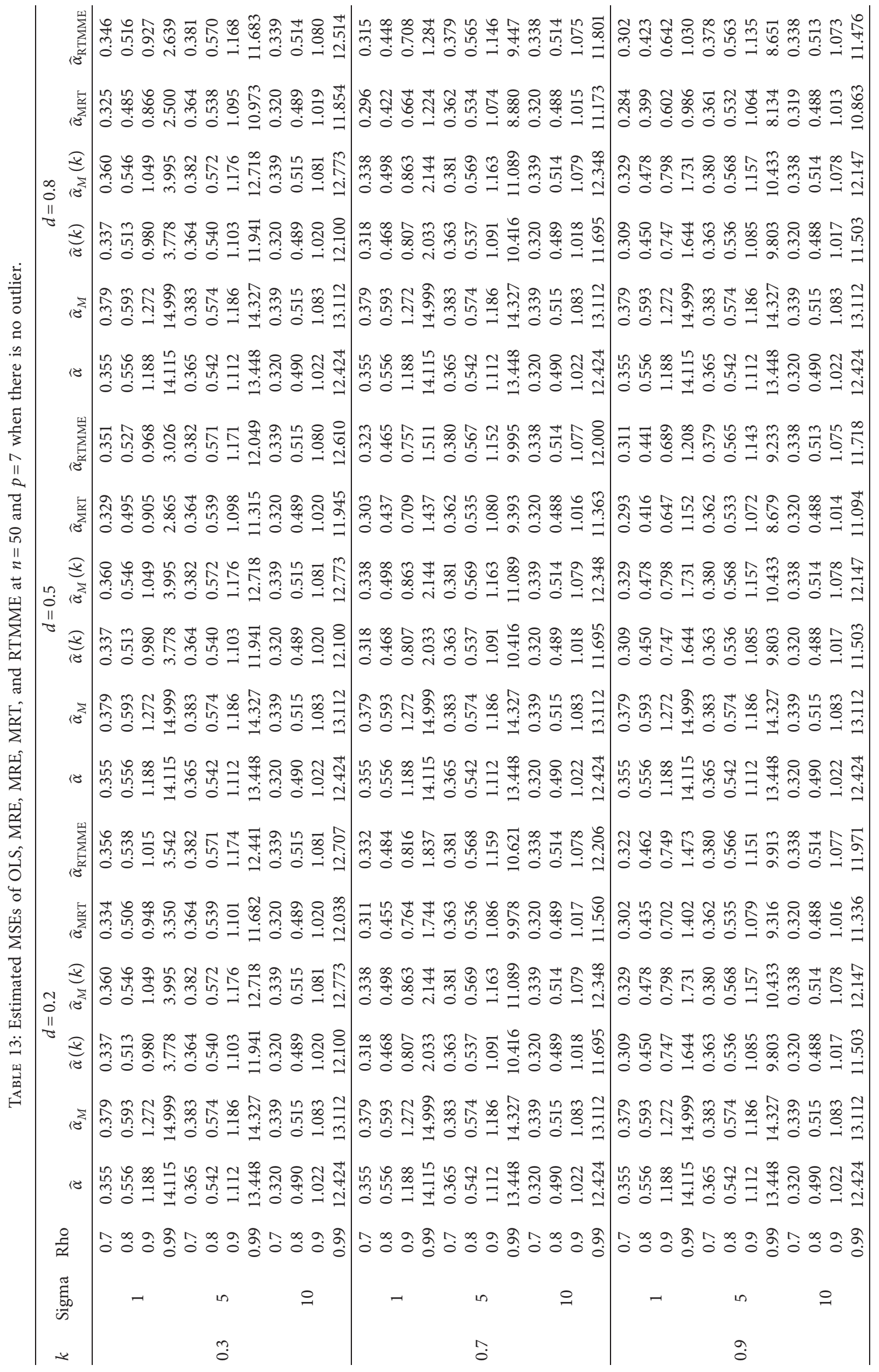




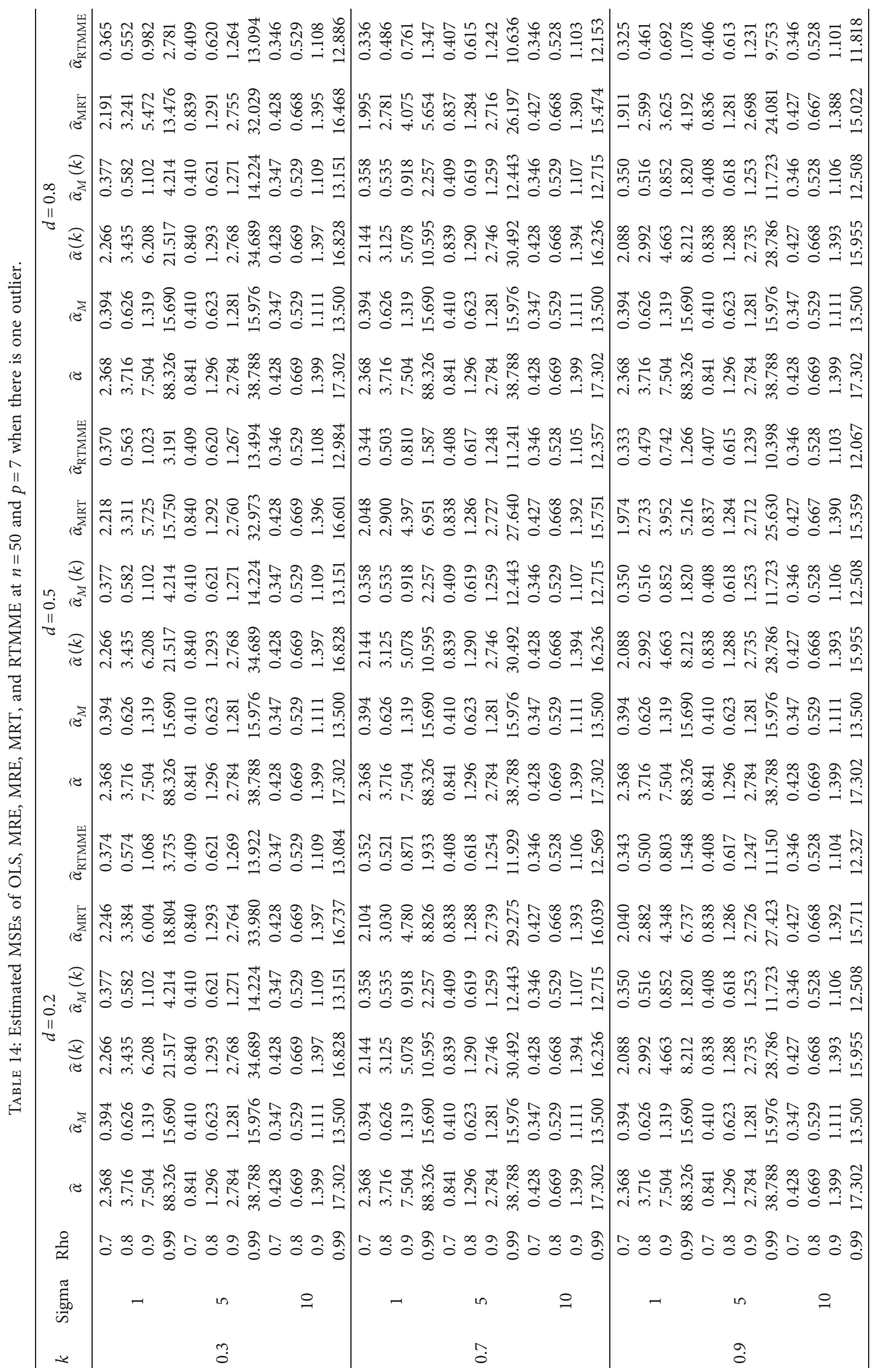




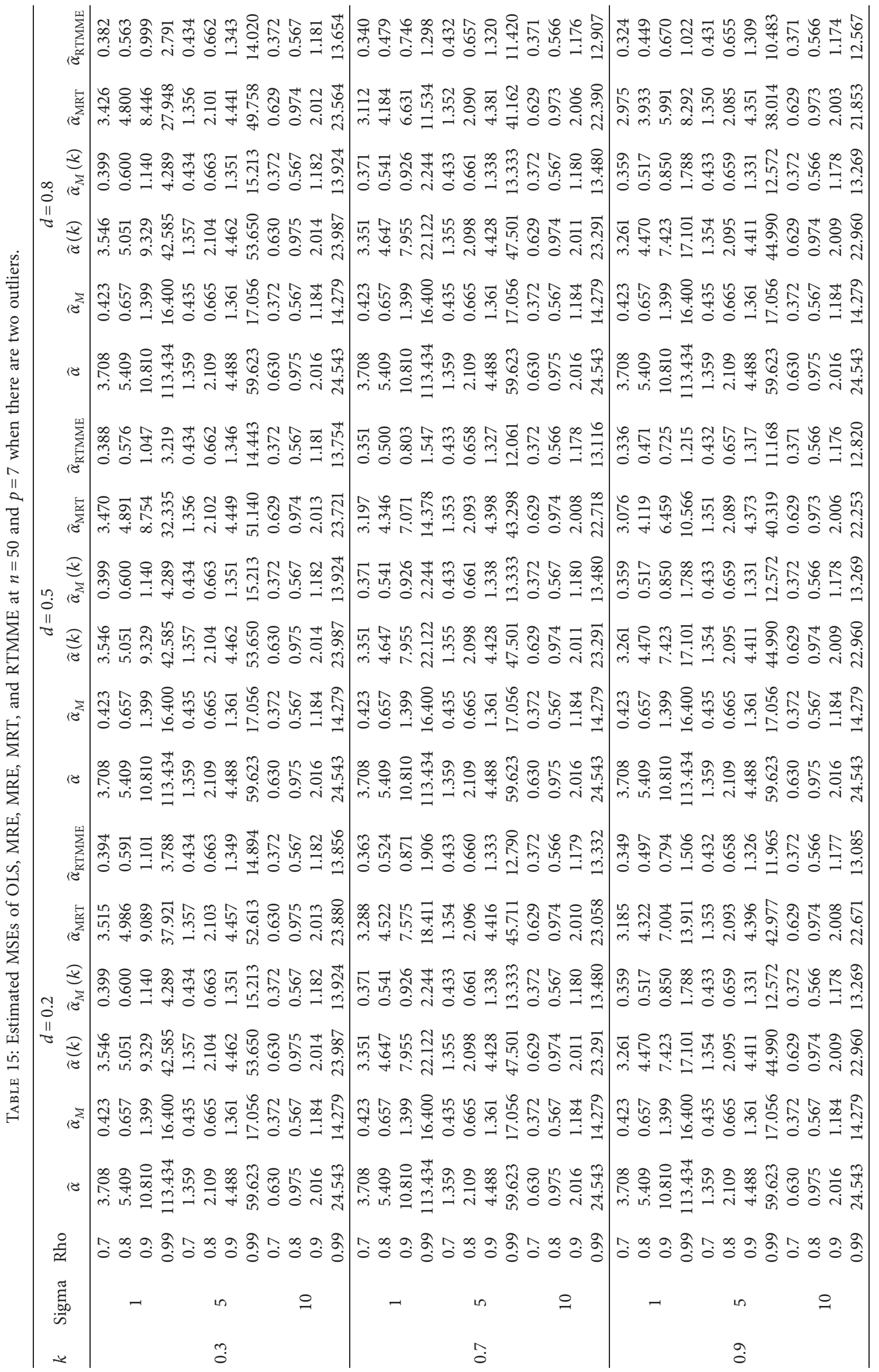




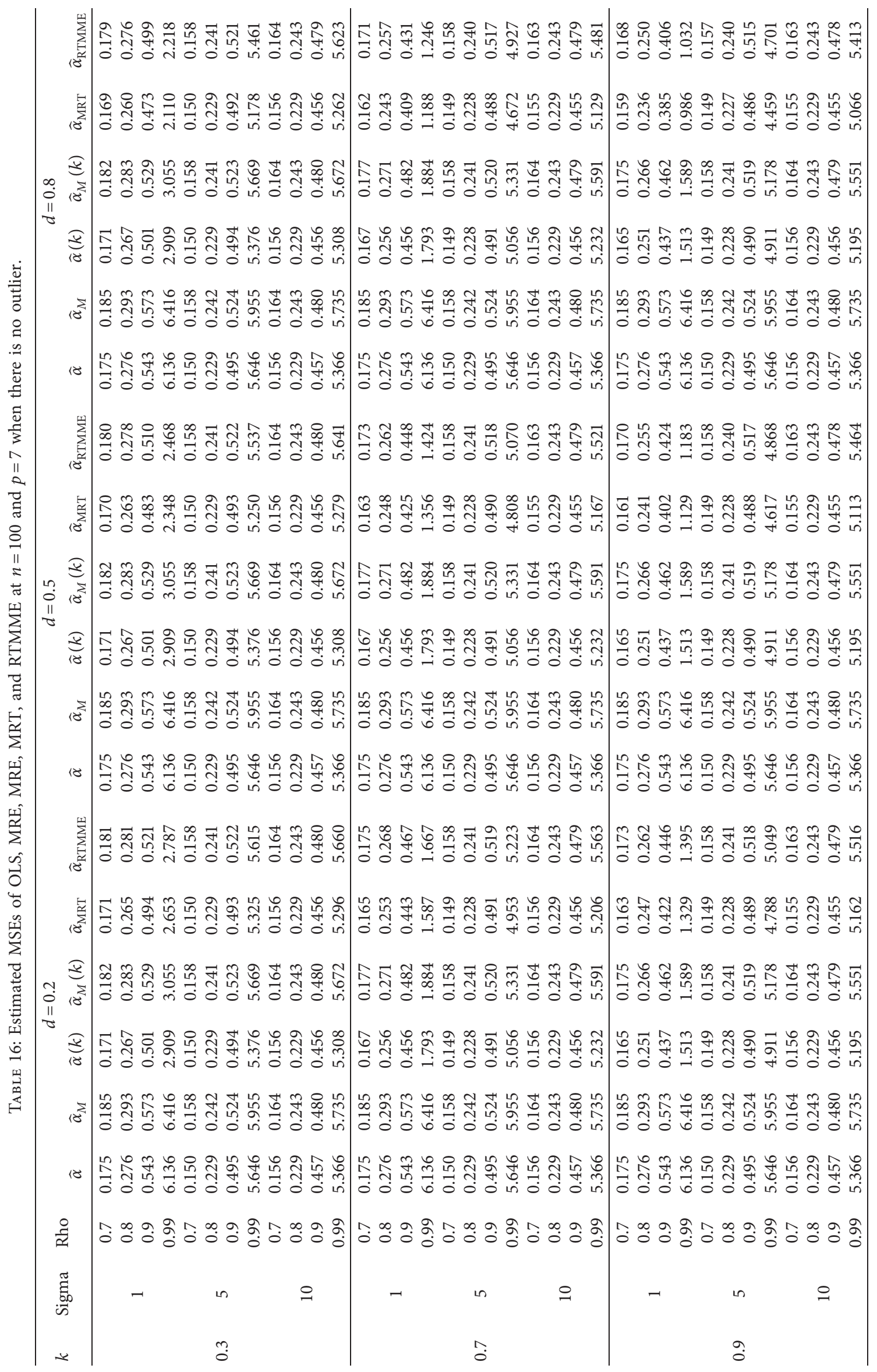




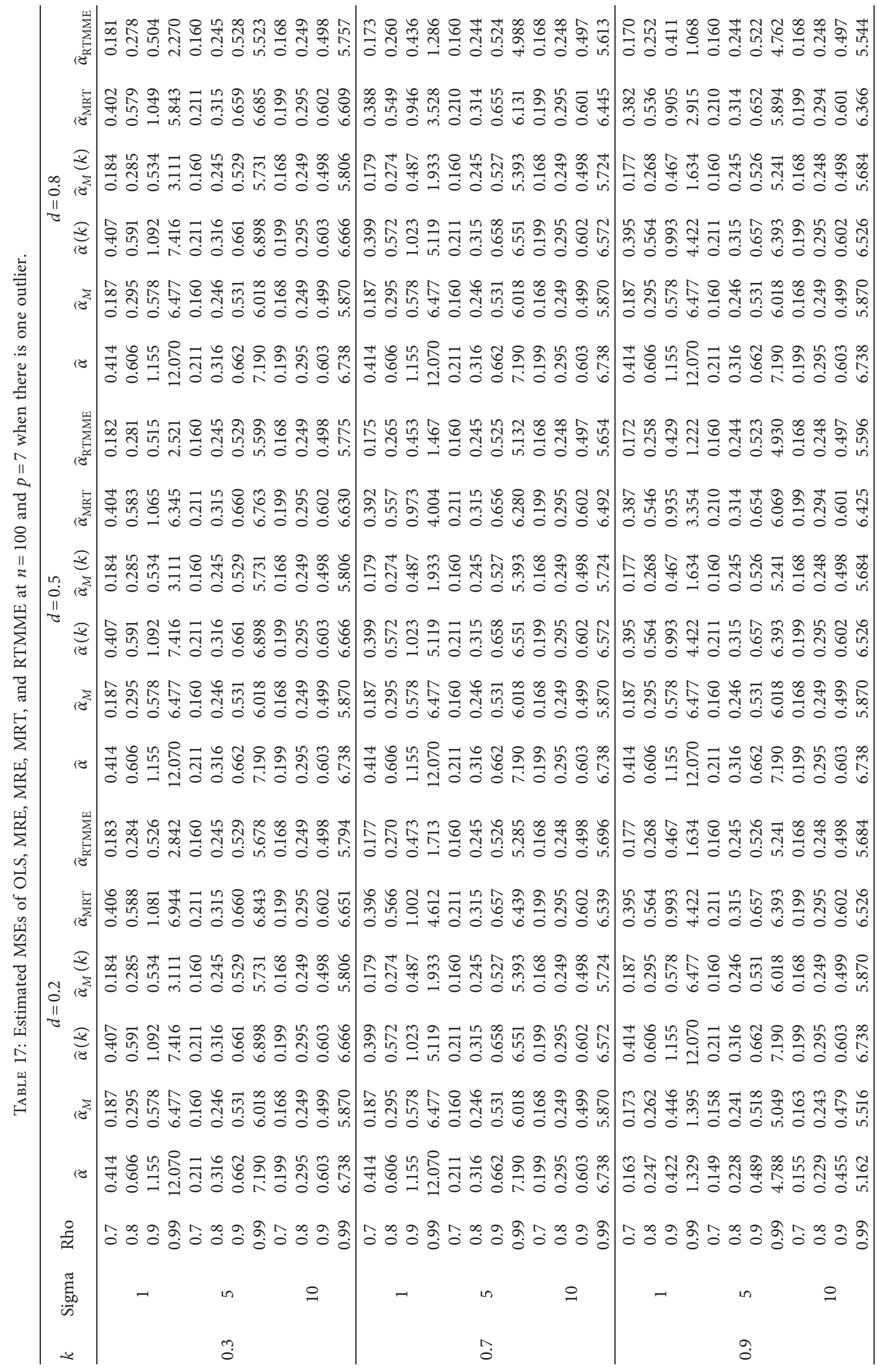




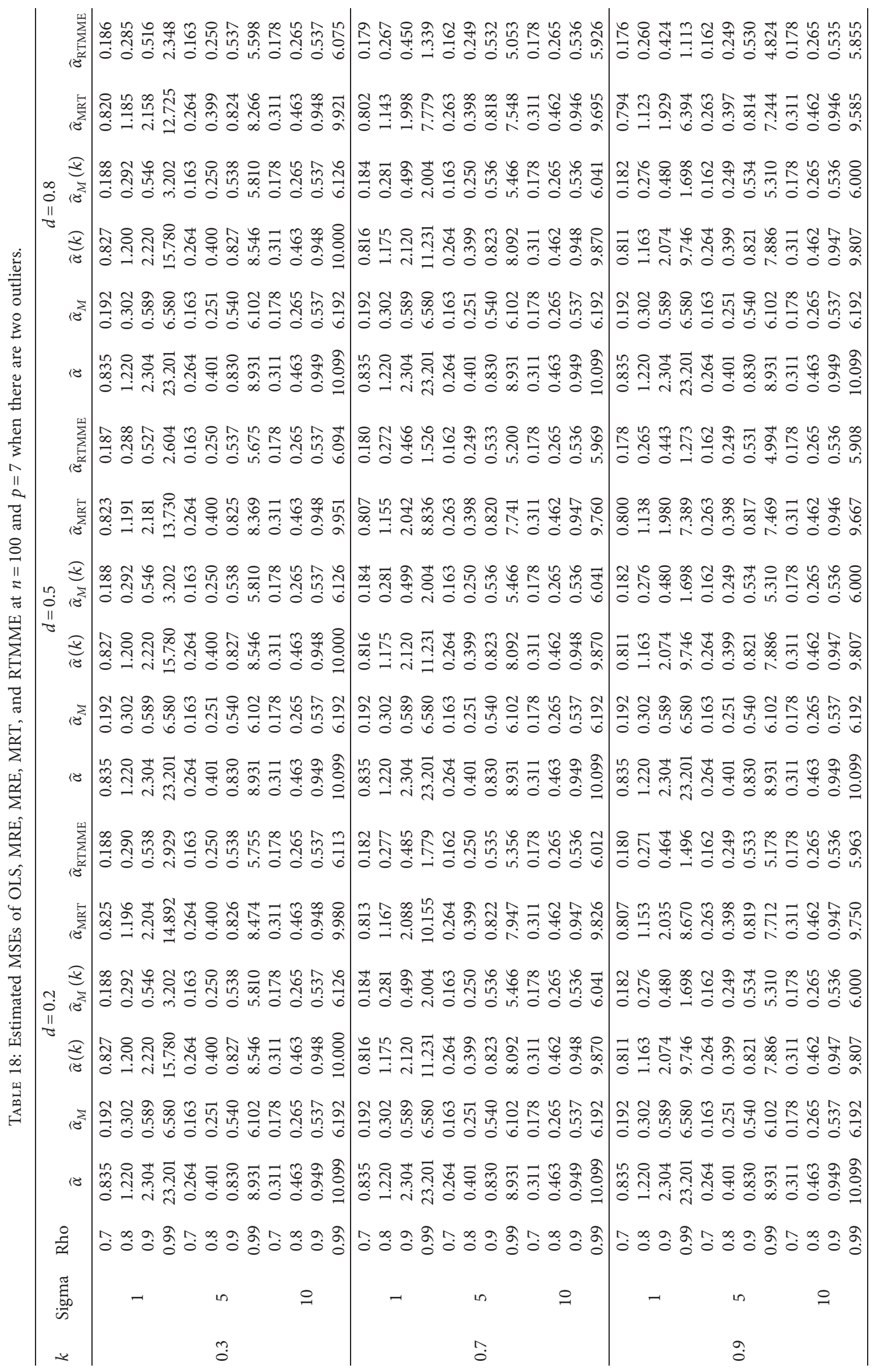


TABLe 19: Estimated MSE values of the OLSE, ME, RE, RME, MRT, and RTMME in Hussein data set.

\begin{tabular}{lcccccc}
\hline Coefficients & $\alpha$ & $\widehat{\alpha}_{M}$ & $\widehat{\alpha}(k)$ & $\widehat{\alpha}_{M}\left(k_{\mathrm{HM}}\right)$ & $\widehat{\alpha}_{\mathrm{MRT}}\left(k_{\mathrm{HMR}}, d\right)$ & $\widehat{\alpha}_{\mathrm{RTMME}}\left(\left(\tilde{k}_{\mathrm{HMR}}, d\right)\right.$ \\
\hline$\beta_{0}$ & 208.874 & 173.331 & 178.6184 & 155.4684 & 178.6184 & -2.0167 \\
$\beta_{1}$ & -2.0171 & -1.8846 & -2.0167 & -1.8844 & 1.5153 & -1.8844 \\
$\beta_{2}$ & 1.51533 & 1.6340 & 1.5153 & 1.6339 & -1.3143 & 1.6339 \\
$\beta_{3}$ & -1.3143 & -1.3527 & -1.3143 & -1.3527 & 2269.33 & -1.3527 \\
MSE & 20875.62 & 9751.86 & 2269.33 & 1952.60 & 629.58 \\
\hline
\end{tabular}

$k=1.9136, k_{\mathrm{HM}}=1.2980, k_{\mathrm{HMR}}=0.9813, k_{\mathrm{HMR}}=0.6656$, and $d=0.95$.

\section{Numerical Example}

Hussein data were originally adopted by Hussein and Zari [18] and later by Lukman et al. [19, 20]. The data contain 31 observations and three explanatory variables. The variance inflation factor for the three explanatory variables is greater than 10 (VIF> 10), which indicates multicollinearity. Lukman et al. [20] identified the following observations as outliers in the $y$-direction: $12,14,15,16,30$, and 31 . Hence, this data set suffers both problems considered in this study. Table 19 shows that the estimated MSE value of the new estimator, RTMME, is smaller compared to the ridge, $\mathrm{M}$-ridge, and MRT estimator.

The theoretical results in this study are validated through the Hussein data as follows:

(i) $\sum_{i=1}^{p} \Omega_{i i}=883.55<\sum_{i=1}^{p} \sigma^{2} \lambda_{i}^{-1}=1850.482$; thus, $\operatorname{MSE}\left(\widehat{\alpha}_{\mathrm{RTMME}}(k, d)\right)<\operatorname{MSE}\left(\widehat{\alpha}_{\mathrm{MRT}}(k, d)\right)$ for every $k>0$. By Theorem 1, this implies that the RTMME outperforms the MRT estimator.

(ii) From Theorem 2, $\mathrm{k}_{1 \mathrm{i}}$ is calculated as [0.2470, 0.0520, $0.0519,0.0002]$. For $k>1.2980\left(k>k_{1 i}\right)$, MSE $\left(\widehat{\alpha}_{\mathrm{RTMME}}(k, d)\right)<\operatorname{MSE}\left(\widehat{\alpha}_{M}(k)\right)$.

(iii) The necessary condition from Theorem 3 is also proven from $\sum_{i=1}^{p} \Omega_{i i}<\sum_{i=1}^{p} \sum_{i=1}^{p}\left(k(1+d) \alpha_{i}^{2}\right) /$ $\left(2 \lambda_{i}+k(1+d)\right)=3026.59$ $=>\operatorname{MSE}\left(\widehat{\alpha}_{\text {RTMME }}(k, d)\right)<\operatorname{MSE}\left(\widehat{\alpha}_{M}\right)$.

\section{Conclusion}

We proposed a two-parameter ridge-type modified M-estimator to jointly handle the problem of multicollinearity and outliers in a linear regression model. Theoretically, the new estimator outperforms the existing estimators under certain conditions. The results of the simulation study and numerical example agree with the theoretical findings. A right choice of $k$ and $d$ also produces better estimates using the proposed estimator. Thus, in the presence of multicollinearity and outliers, this estimator can effectively replace the following estimators: the ordinary least squares estimator, the M-estimator, the ridge estimator, the M-ridge estimator, and the modified ridge-type estimator.

\section{Data Availability}

The data used to support the findings of this study are available in page 7 of [19].

\section{Conflicts of Interest}

The authors declare that they have no conflicts of interest.

\section{References}

[1] D. N. Gujarati, Basic Econometrics, McGraw-Hill, New York, NY, USA, 1995.

[2] A. F. Lukman, Classification-Based Ridge, Lambert Academic Publishing, Beau-Bassin, Mauritius, 2018.

[3] A. F. Lukman and K. Ayinde, "Review and Classifications of the ridge parameter estimation techniques," Hacetteppe Journal of Mathematics and Statistic, vol. 46, no. 5, pp. 953967, 2017.

[4] A. E. Hoerl and R. W. Kennard, "Ridge regression: biased estimation for nonorthogonal problems," Technometrics, vol. 12 , no. 1 , pp. 55-67, 1970.

[5] K. Liu, "A new class of biased estimate in linear regression," Communication in Statistics-Theory and Methods, vol. 22, pp. 393-402, 1993.

[6] C. Stein, "Inadmissibility of the usual estimator for mean of multivariate normal distribution," in Proceedings of the Third Berkley Symposium on Mathematical and Statistics Probability, J. Neyman, Ed., vol. 1, pp. 197-206, University of California Press, Berkeley, CA, USA, 1956.

[7] W. F. Massy, "Principal components regression in exploratory statistical research," Journal of the American Statistical Association, vol. 60, no. 309, pp. 234-256, 1965.

[8] B. F. Swindel, "Good ridge estimators based on prior information," Communications in Statistics - Theory and Methods, vol. 5, no. 11, pp. 1065-1075, 1976.

[9] P. J. Huber, Robust Statistics, Wiley, New York, NY, USA, 1981.

[10] M. J. Silvapulle, "Robust ridge regression based on an M-estimator," Australian Journal of Statistics, vol. 33, no. 3, pp. 319-333, 1991.

[11] K. Ayinde, A. F. Lukman, and O. T. Arowolo, "Robust regression diagnostics of influential observations in linear regression model," Open Journal of Statistics, vol. 5, pp. 1-11, 2015.

[12] A. V. Dorugade, "Adjusted ridge estimator and comparison with Kibria's method in linear regression," Journal of the Association of Arab Universities for Basic and Applied Sciences, vol. 21, no. 1, pp. 96-102, 2016.

[13] A. F. Lukman, K. Ayinde, S. Binuomote, and O. A. Clement, "Modified ridge-type estimator to combat multicollinearity: applications to chemical data," Journal of Chemometrics, vol. 33, no. 5, p. e3125, 2019.

[14] F. R. Hampel, E. M. Ronchetti, P. J. Rousseeuw, and W. A. Stahel, Robust Statistics. The Approach Based on Influence Function, Wiley, New York, NY, USA, 1986. 
[15] B. M. G. Kibria, "Performance of some new ridge regression estimators," Communication in Statistics-Simulation and Computation, vol. 32, no. 1, pp. 419-435, 2003.

[16] G. C. McDonald and D. I. Galarneau, "A Monte Carlo evaluation of some ridge-type estimators," Journal of the American Statistical Association, vol. 70, no. 350, pp. 407-416, 1975.

[17] A. F. Lukman, K. Ayinde, and A. S. Ajiboye, "Monte Carlo study of some classification-based ridge parameter estimators," Journal of Modern Applied Statistical Methods, vol. 16, no. 1, pp. 428-451, 2017.

[18] Y. Hussein and M. Zari, "Generalized two stage ridge regression estimator TR for multicollinearity and autocorrelated errors," Canadian Journal on Science and Engineering Mathematics, vol. 3, no. 3, pp. 79-85, 2012.

[19] A. F. Lukman, K. Ayinde, S. K. Sek, and E. Adewuyi, "A modified new two-parameter estimator in a linear regression model," Modelling and Simulation in Engineering, vol. 2019, Article ID 6342702, 10 pages, 2019.

[20] A. F. Lukman, O. I. Osowole, and K. Ayinde, "Two stage robust ridge method in a linear regression model," Journal of Modern Applied Statistical Methods, vol. 14, no. 2, pp. 53-67, 2015. 